\title{
Comparative Fluid-Structure Interaction Analysis on Influence of Banding Conditions to Ascending Aorta Aneurysm Formation in Rat
}

\section{Juan Su}

China Medical University https://orcid.org/0000-0002-1887-3622

Jun Fan

China Medical University

Qing Tang

China Medical University

\section{Shijie Chang}

China Medical University

Xianzheng Sha ( $\nabla$ xzsha@cmu.edu.cn )

China Medical University https://orcid.org/0000-0002-1083-7862

\section{Research}

Keywords: Ascending aortic aneurysm, Banding, Fluid-structure interaction, Rat

Posted Date: October 14th, 2020

DOl: https://doi.org/10.21203/rs.3.rs-89400/v1

License: (9) This work is licensed under a Creative Commons Attribution 4.0 International License. Read Full License 


\section{Abstract}

Background: Ascending aortic aneurysm in an important cause of mortality in cardiovascular diseases. Stenosis of aortic is considered to be a risk factor as the ascending aortic aneurysm grows. Animal models have been demonstrated that ascending aortic aneurysm could be induced by supra valvular banding of the ascending aortic. Our objective is to compare different banding conditions on the formation of aneurysms for more precise experiment and improving the preclinical value. Therefore, three comparison banding groups of banding altitude, banding severity and banding angle are established based on rat. Then flow pattern, wall shear stress (WSS) and vessel deformation of each model are calculated and discussed using transient two-way fluid-structure interaction (FSI) analysis in order to explore the influence of different banding methods on the generation of ascending aorta aneurysm.

Results: Banding methods lead to different shapes or amplitudes of flow beam, WSS and vessel dilation. Eccentric flow beam, local high WSS accompany with vessel dilation are formed above the banding ring in all banding models because of the banding operation compared with normal model. More concentrated flow beam with bigger velocity, higher local WSS and more obvious expansion deformation above the banding ring are prone to happen in the middle segment banding with $60 \%$ banding severity and banding angle of 30 degree.

Conclusion: According to the results, a higher position, relatively severe banding, and an acute banding angle are more favor to promote the generation of ascending aortic aneurysm.

\section{Background}

Aortic aneurysm is a potentially dangerous disease that often causes death by dissection or rupture [1] in general, people with aortic stenosis or bicuspid aortic valve (BAV) are considered to have an increased risk of developing an ascending aortic aneurysm[2-4].Many studies attribute this phenomenon to abnormal hemodynamic factors caused by stenosis in addition to genetic effects[5-8].

Animal models have been successfully made to study the pathogenesis of aneurysm caused by aortic stenosis [9-12], effectively obtaining longitudinal features of aneurysm than from individuals. Models in pig [11] and our previous experiment in rat [12] showed that ascending aortic aneurysm could be induced by banding supra valvular of the ascending aortic. These models went through a chronic process which had a similarity to a certain extent with clinical ascending aorta aneurysm formation accompanied by aortic stenosis or BAV, indicating a delightful underlying clinical value. Banding played a key role in leading to the coarctation of the aorta and banding methods determined the different states of aorta stenosis during the experiments. Patient-specific studies demonstrated that different stenosis states could affect the formation of aneurysms. Schaefer et al found that valve opening orientation has been associated with the rate of ascending aortic dilation $[13,14]$. Although there were conflicts in relationship between stenosis severity and ascending aorta aneurysm, Alessandro et al indicated that mid-ascending dilatation was proportional to stenosis severity [15]. Alexia et al acknowledged that the severity of aortic 
stenosis was correlated to the progression of aortic dilatation [16].However, banding methods that may largely affect the formation of aneurysms has not been discussed yet. A clear understanding of these events is critical for mechanistic exploitation in ascending aortic aneurysm. Therefore, different banding methods are worth comprehensive screening.

Since computational fluid dynamics (CFD) represents a promising method which performed with notable advantages such as great convenience, high speed and effective acquisition of additional hemodynamic parameters. It is widely accepted in analyzing the correlation between aortic pathology and hemodynamic changes, predicting and ameliorating the understanding of disease progression [17].

Therefore, taking these issues into account, the current study discusses the impact of three banding conditions of altitude, angle and severity on the formation of ascending aortic aneurysms based on numerical simulation of two way transient fluid-structure interaction by using finite element method, which can serve for precision experiment and improve the preclinical value of aortic aneurysm research.

\section{Results}

Velocity, WSS and wall displacement (with the same scale) were obtained in all models at the time of $0.23 \mathrm{~s}$ in the acceleration segment of systolic phase. Hemodynamic changes, especially between banding ring and brachiocephalic, where the ascending aortic aneurysm formed (we denoted this zone to be the region of interested (ROI)), were contrasted and analyzed during each group. ' $A$,', $B$ ', 'C' points in Fig. 3(a), Fig. 5(a), Fig. 7(a)are on behave of typical regions of under banding ring(A),flow impinging region(B) and descending aorta(C). WSS and displacement data are extracted along the outer edge of ascending aorta as indicated in red line in Fig. 3 (b-1) in each group.

\section{Comparison of models with different banding altitudes}

The profile of velocity streamline in normal model is uniform and parallel along the aortic wall (Fig. 3(a1)). Flow beam is generated after blood flowing through the banding ring, impinging against the convex wall of ascending aorta due to the curvature in the origination of aorta arch and then deflects to the direction of the aortic arch. This flow beam is less converged in proximal segment model than that in middle segment one (Fig. 3 (a-2), Fig. 3(a-3)). Average velocities in regions of A, B and C are showed in Fig. 4(a). There is an obviously higher value in position $B$ in middle segment banding model. However, average velocities in position $A$ and $C$ is quite similar no matter with banding or banding altitude.

Higher WSS can be observed in aortic arch in normal model (Fig. 3(b-1)) and in the area adjacent to flowimpinging zone in ROI in banding models(Fig. 3(b-2) and(b-3)). Dilation is obvious in banding models both above and under banding ring(Fig. 3(c-2)(c-3)). Furthermore, in the ROI, there is more evident dilation in middle banding model than that in proximal banding model. We can see that WSS and displacement of ROI in middle segment model is larger than that in proximal segment and normal models(Fig. 4(b)and (c)). 
Similar with banding altitude group, Fig. $5((\mathrm{a})(\mathrm{b})(\mathrm{c})$ )show the velocity streamline, WSS contours and displacement contours of deformation, respectively, and we can see banding severity of $40 \%, 50 \%, 60 \%$ models in the columns.

In the ROI, it is legible that more concentrated velocity streamline exists in the model of banding severity of $60 \%$ with a larger velocity value of about $0.7 \mathrm{~m} / \mathrm{s}$ at position $B$ in the impinging zone. Blood flow velocities in three models at position $A$ and $C$ are nearly the same (Fig. 6(a)). Meanwhile, high WSS and dilation can be seen adjacent to the flow-impinging zone (Fig. 5(b)(c)), and the magnitudes of WSS and this dilation are becoming more obviously from $40-60 \%$ severity model. Based on the WSS and displacement curves in Fig. $6((\mathrm{~b})(\mathrm{c})$ ), we can also see that WSS and displacement above the banding ring elevate with the increasing of banding severity.

\section{Comparison of models with different banding angles}

Velocity streamline, WSS contours and displacement with deformation are showed in Fig. 7, among whose columns represent models of banding angle of 0 degree, 30 degree and 150 degree, respectively. Different flow jet trajectories can be seen in Fig. 7(a) because of banding angles: there is no obvious impinging zone in model of 150 degree compared with other two models in ROI. The impinging zone in 0 degree model locates at the aorta arch, while the corresponding zone in 30 degree model is slightly higher than the banding ring with a shorter distance. The 30 degree model has the biggest value of velocity in $\mathrm{B}$ position. The velocities in A and C positions are in the likelihood in all three models (Fig. 8(a)).

High WSS, in the ROI, in models of 0 degree and 30 degree situates on the convex wall of ascending aorta while high WSS locates along the inner wall in 150 degree models. The deformation in Fig. 7(c) shows that expansion is more prone to happen in 30 degree model than in 0 degree model whereas retraction of the aorta can be seen in model of 150 degree. In Fig. 8(b) and (c), we can also find that model of 30 degree have significant larger value in WSS and displacement above the banding ring than other two models.

\section{Discussion}

Banding the ascending aorta is a practical method in simulating aortic stenosis in experiment animals, contributing to reveal the relationship between hemodynamic characteristics and pathological features, helping us enhance our flexible understanding of aneurysm [18]. In current study, three different banding factors of altitude, severity and degree are discussed for predicting the impact on the formation of ascending aortic aneurysms based on a rat. It is valuable, for one hand, since the cardiovascular system of the rat has a lot of similarity to that of human; for another hand, it's very difficult to collected invasive clinical disease data from humans in most cases, thus, animal model becomes a preferable way for producing predictable and controllable symptoms $[9,19]$. 
In this study, two basic but critical hemodynamic risk factors that of flow pattern together with WSS, which can significantly promote the initiation of ascending aorta aneurysm are calculated. Eccentric systolic flow, displaced from the centerline toward the vessel wall, is one of a topic of clinical interest in exploring the role of rheology in the formation of ascending aortic aneurysm. Researchers have reported that stronger eccentric blood flow jet toward the aortic wall, which may include higher velocity, significant flow displacement and jet-to-wall impingement, lead to vascular remodeling and aneurysm formation [11, $20,21]$. Ayaon-Albarran et al [11] found aortic wall that was directly hit by eccentric flow jet were thinner, given that decreased thickness in vessel wall could result in aortic dissection. Cebral et al[22, 23] considered that a concentrated flow beam may cause an intracranial aneurysm to progress, and the decrease of the flow impingement size is more likely to cause aneurysm to experience growth or rupture than large impingement one. Moreover, in the context of flow jet, WSS levels are considered abnormally high adjacent to the flow impingement zone. This high WSS has long been accepted as a dangerous motivational factor predisposing a vessel wall to aneurysm initiation and development, which behave in early smooth muscle cell apoptosis or changes to extracellular matrix protein expression [2426].Evidence from Guzzardi's study showed that elastin fibers in regions of elevated WSS were significantly thinner compared with normal ones[27].Clinical data also supported ascending aorta dilatation occur in regions of elevated WSS values[28]. Displacement simulation provides us intuitive view of expansion of the vessel, indicating the suspicious location of the potential lesion. In most studies of vascular deformation, they pay more attention to the existed condition of aneurysm or its annual growth rate for the purpose of predicting rupture[29], lacking of comparison deformation information in a cardiac cycle under the background of non-aneurysm status. Rat experiment in intracranial aneurysm of Koseki et al [30] indicated that high WSS together with local vessel transient outward bulging determined the prospective site of aneurysm formation. We can see clearly that the relative angle and distance between the stenosis plane and the aortic curvature result in different flow jet forms, WSS distributions and vessel deformation above the banding ring. Therefore, combining these three calculation results, we have reasons to speculate that a higher position, relatively severe banding, and an acute banding angle are more inclined to promote the formation of ascending aortic aneurysm.

We also find an obvious large deformation under the banding ring. This unique apace was created due to supra valvular banding of the aortic. Different with the dilation above the banding ring, velocity and WSS are changeless in this region. It is thought to be associated with increased pressure load caused by the outflow tract obstruction [31]. This type of force may play a key role in remodeling of aortic wall thickness rather than adjusting diameter of the aorta in early stage [32].

There are limitations in this study. Like some methodology of simulation, a few ideal hypotheses are adopted here, especially the morphological structure of rat's aorta. However, we believe that this kind of ideal structure is universal and commonly used for simulation calculations. As examples, Pierre et al [32] modeled mouse carotid artery as a cylinder, Gelide et al[34]simulated physiological geometry of the descending aorta with a funnel-like structure. Therefore, we think our simulation is acceptable under the background of comparison. 
In summary, this study showed for the first time, how the ascending aortic aneurysm can be induced with different banding conditions, which will do favor to promote the generation of ascending aortic aneurysm. This promising finding warrant further mechanistic investigation into the formation of aortic aneurysm.

\section{Conclusions}

Related animal models are indispensable tools in obtaining longitude features of aneurysm than from individuals; therefore, it is necessary to make the experiment more efficient. Under the background of these banding-reduced aneurysm animal models, the banding method is a problem that cannot be ignored in affecting the growth of aneurysms.

In this paper, we modeled the ascending aorta banding operation in rat and three banding profiles of banding altitude, banding severity, banding angle were established, separately. For each banding group, blood flow patterns, WSS distributions and vessel deformation are calculated and compared. Our study suggests that banding methods could obviously produce differences in flow velocity, concentration and eccentricity of flow beam and local high WSS which are considered to have a significant influence on aneurysm formation. A higher position, relatively severe banding, and an acute banding angle are more favor to promote the generation of ascending aortic aneurysm.

\section{Methods}

Three-dimensional aorta geometries with different banding patterns were created based on morphology feature of ascending aorta in rat using Ansys Designmodeler (Fig. 1). The fundamental aortas starting from aortic root were the same in shape and size for all models, including ascending aorta(AA), aortic arch with three primary branches (brachiocephalic (BCA),left common carotid (LCCA) and left subclavian artery (LSCA)) and descending aorta. The value of $D_{A}$ was 2.3mm[12]. $D_{B}, D_{L C}, D_{L S}, R_{A}$ and $L_{A}$ were set to be $1.2 \mathrm{~mm}, 1.1 \mathrm{~mm}, 1.1 \mathrm{~mm}, 4.5 \mathrm{~mm}$ and $6 \mathrm{~mm}$ respectively (Fig. 1(a)). The aortic wall was assumed uniform with the thickness of $0.15 \mathrm{~mm}$.

Constriction was used to represent the banding position. Banding severity was calculated by $\left(D_{A}-D_{C}\right) / D_{A}$ (Fig. 1f).Banding angle $(\theta)$ was defined by included angle between aorta inlet plane and banding ring plane (Fig. 1d and e). All of models were divided into three groups for comparison purpose as illustrated in Table1. 
Table 1

Groups and compositions for models

\begin{tabular}{|ll|}
\hline groups & Compositions \\
\hline banding altitude & normal model \\
(banding angle 0 degree, banding severity of 60\%) & $\begin{array}{l}\text { proximal segment banding model } \\
\text { middle segment banding model }\end{array}$ \\
\hline banding severity & banding severity of $40 \%$ \\
(middle segment banding, banding angle 0 degree) & banding severity of $50 \%$ \\
\hline banding angle & banding severity of $60 \%$ \\
(middle segment banding, banding severity of 60\%) & banding angle of 0 degree \\
& banding angle of 30 degree \\
\hline
\end{tabular}

Blood was modeled to be homogeneous, incompressible, adiabatic Newtonian fluid with density of $1.06 \mathrm{~kg} / \mathrm{m}^{3}$ [9] and viscosity of $0.004 \mathrm{pa}[\mathrm{s}$ [9]. A longitudinal velocity was applied at the aorta root as given by Fig. 2, in which the heart rate of the rat was estimated at approximately 300 beats per second [9] and the peak flow velocity of was about $0.6 \mathrm{~m} / \mathrm{s}$ [35]. At the outlet of BCA, LCCA, LSCA and DA, a constant reference pressure was given to be zero. Although it is not a real-data boundary condition, this is a reasonable simplification on account of comparison purpose in this study without ignoring main blood characters of pulsatile flow.

Vessel wall was assumed to be isotropic elasticity. Young's modulus of aorta was set to be $7.5 \times 10^{5} \mathrm{~Pa}$ according to the research of Guo and Band [36, 37]. Possion's ratio and density were $0.45[9]$ and $1.06 \times$ $10^{3} \mathrm{~kg} / \mathrm{m}^{3}[38]$ respectively. The fluid-solid interface was set at the interface of blood and vessel wall, and fixed constraint was given at inlet or outlet of aorta.

Blood and vessel wall were meshed separately. In the geometry of blood, 'inflation' was set to have maximum layers of 5, growth rate of 1.2 and the division in edge of ascending inlet was 15 . Then, in the vessel wall, to match a good numeric resolution, the division in the edge of ascending wall was also set to be 15, at the same time, refinement and explicit in physics preference was used to help to obtain grids of good quality. In total, there were about 50 thousand elements in blood geometry and about 35 thousand elements in vessel wall geometry in all models due to similarity in their shapes and dimensions. This kind of mesh was small enough to satisfy iteration convergence in blood-wall interface.

Ansys workbench (ANSYS Inc, Canonsburg, USA) was used to solve the transient two-way fluid-structure interaction simulation with second order back ruler method for time integration. Three cardiac cycles were performed to improve the precision of calculation with a time step of $0.005 \mathrm{~s}$. The second cycle was extracted for post processing. 


\section{Abbreviations}

WSS

wall shear stress

CFD

computational fluid dynamics

FSI

fluid-structure interaction

BAV

bicuspid aortic valve

BCA

brachiocephalic

LCCA

left common carotid

LSCA

left subclavian artery

$\mathrm{ROI}$

region of interested

\section{Declarations}

Ethics approval and consent to participate

Not applicable.

Consent for publication

Not applicable.

Availability of data and materials

All data generated or analyzed during this study are included in this published article.

\section{Competing interests}

The authors declare that they have no competing interests.

\section{Funding}

This study was supported by Scientific Research Fund of Liaoning Provincial Education Department (LQNK201744), Natural Science Foundation of Liaoning Province (2018055003).

\section{Acknowledgements}


The authors are grateful to all study participants and give my sincere thanks to associate professor Xiran Jiang for his kind help.

\section{Author's Contributions}

JS implemented the calculation and analyzed the results, and was a major contributor in writing the manuscript. JF suggested the banding conditions and provided the guidance. QT gave help in calculation and data analysis. ShjC consulted the obtained results. XzS provided the guidance and consulted the obtained results. All authors have read an approve the final manuscript

\section{References}

[1]Isselbacher EM.Thoracic and abdominal aortic aneurysm.Circulation.2005;111:816-28. http://dx.doi.org/ 10.1161/01.CIR.0000154569.08857.7A

[2] Tadrod TM, Klein MD, Shapira OM. Ascending aortic dilatation associated with bicuspid aortic valve: pathophysiology, molecular biology, and clinical implications. Circulation. 2009;119:880-890. http://dx.doi.org/ 10.1161/CIRCULATIONAHA.108.795401

[3]Goubergrits L,Mevert R,Yevtushenko P, et al.The impact of MRI-based inflow for the hemodynamic evaluation of aortic coarctation. Annal of Biomedical Engineering.2013;41(12):2575-2587. http://dx.doi.org/ 10.1007/s10439-013-0879-2

[4]Kerrneis C,Pasi N,Arangalage D,et al. Ascending aorta dilatation rates in patients with tricuspid and bicuspid aortic stenosis: the COFRASA/GENERAC study. European Heart Journal-Cardiovascular Imaging.2017; 0:1-8. http://dx.doi.org/ 10.1093/ehjci/jex176

[5] Andersson M, Lantz J, Ebber T, Karlsson M. Quantitative assessment of turbulence and flow eccentricity in an aortic coarctation: impact of virtual interventions. Cardiovascular Engineering and Technology.2015;6(3):281-293. http://dx.doi.org/ 10.1007/s13239-015-0218-x

[6]Faggiano E, Antiga L, Puppini G,et al. Helical flows and asymmetry of blood jet in dilated ascending aorta with normally functioning bicuspid valve. Biomech Model Mechanobiol.2013; 12:801-813. http://dx.doi.org/ 10.1007/s10237-012-0444-1

[7] Cao K,Sucosky P. Effect of bicuspid aortic valve cusp fusion on aorta wall shear stress:prelimnary computational assessment and implication for aortic dilation. World Journal of Cardiovascular Diseases.2015; 5:129-140. http://dx.doi.org/ 10.4236/wjcd.2015.56016

[8] Bonomi D, Vergara C, Faggiano E,et al. Influence of the aortic valve leaflets on the fluid-dynamics in aorta in presence of a normally functioning bicuspid valve. Biomech Model Mechanobiol. 2015; 14:1349-1361. http://dx.doi.org/ 10.1007/s10237-015-0679-8 
[9] Al-Rawi M, Al-Jumaily AM. Assessing abdominal aorta narrowing using computational fluid dynamics. Med Biol Eng Comput.2016;54:843-853. http://dx.doi.org/ 10.1007/s11517-015-1375-7

[10] Park H, Park JH ,Lee SJ. In vivo measurement of hemodynamic information in stenosed rat blood vessels using X-ray PIV. Scientific Reports.2016;6:37985 . http://dx.doi.org/ 10.1038/srep37985

[11] Albarran AA, Jimenez RF, Guisasola JS,et al. Systolic flow displacement using 3D magnetic resonance imaging in an experimental model of ascending aorta aneurysm:impact of rheological factors. European Journal of Cardio-Thoracic Surgery.2016;50(4):685-

692.http://dx.doi.org/10.1093/ejcts/ezw132

[12]Fan J, Li X, Zhong LL, et al. MCP-1, ICAM-1 and VCAM-1 are present in early aneurysmal dilatation in experimental rats. Folia Histochem Cytobiol. 2010;48(3): 455-461. http://dx.doi.org/ 10.2478/v10042010-0042-y

[13]Schaefer BM,Lewin MB,Stout KK,et al.The bicuspid aortic valve:an intergrated phenotypic classification of leaflet morphology and aortic root shape.Heart. 2008;94(12):1634-1638. http://dx.doi.org/ 10.1136/hrt.2007.132092

[14]Thanassoulis G,Yip JWL,Filion K,et al.Retrospective study to identify predictors of the presence and rapid progression of aortic dilatation in patients with bicuspid aortic valve.Nat Clin Pract Cardiovasc Med.2008;5(12):821-8. http://dx.doi.org/ 10.1038/ncpcardio1369

[15] Corte AD, Bancone C, Quarto C,et al. Predictors of ascending aortic dilatation with bicuspid aortic valve:a wide spectrum of disease expression. European Journal of Cardio-thoracic Surgery.2007; 31(3):397-404. http://dx.doi.org/ 10.1016/j.ejcts.2006.12.006

[16] Rossi A, van der Linder D,Yap SC,et al. Ascending aorta dilatation in patients with bicuspid aortic valve stenosis: a prospective CMR study. Eur Radiol.2013;23(3):642-649. http://dx.doi.org/ $10.1007 / \mathrm{s} 00330-012-2651-7$

[17]Ong CW,Wee I,Syn Nicholas,et al. Computational fluid dynamics modeling of hemodynaomic parameters in the human diseased aorta: a systematic review. Ann Vasc Surg.2020; 63: 336-381. http://dx.doi.org/ 10.1016/j.avsg.2019.04.032.

[18] Galis ZS,\& Khatri J J. Matrix metalloproteinases in vascular remodeling and atherogenesis the good, the bad, and the ugly. Circ. Re.2002;22(90):251-262. http://dx.doi.org/ 10.1161/res.90.3.251

[19]Vukicevic AM,Stepanovi NM,Jovicic GR,et al. Computer methods for follow-up study of hemodynamic and disease progression in the stented coronary artery by fusing IVUS and X-ray angiography. Med Biol Eng Comput.2014;52(6):539-556. http://dx.doi.org/10.1007/s11517-014-1155-9

[20]Viscardi F, Vergara C, Antiga L, et al. Comparative finite element model analysis of ascending aortic flow in bicuspid and tricuspid aortic valve. Artificial Organs.2010;34(12):1114-1120. http://dx.doi.org/ 
[21] Menon A, Wendell DC, Wang HF, et al. A coupled experimental and computational approach to quantify deleterious hemodynamics, vascular alterations, and mechanisms of long-term morbidity in response to aortic coarctation. Journal of Pharmacological and Toxicological Methods.2012;65(1):1-28. http://dx.doi.org/ 10.1016/j.vascn.2011.10.003

[22] Cebral JR, Sheridan M,Putman CM.Hemodynamics and bleb formation in intracranial aneurysms.AJNR Am J Neuroradiol.2010;31(2): 304-310. http://dx.doi.org/ 10.3174/ajnr.A1819

[23] Cebral JR,Castro MA, Burgess JE,et al. Characterization of cerebral aneurysms for assessing risk of rupture by using patient-specific computational hemodynamics models.AJNR Am J Neuroradiol. 2005; 26(10):2550-2559. http://dx.doi.org/10.1080/02841850500216293

[24]Dolan JM,Meng $H$,Singh $H$,et al.High fluid shear stress and spatial shear stress gradients affect endothelial proliferation,survival,and alignment.Annals of biomedical engineering.2011; 39(6): 16201631. http://dx.doi.org/ 10.1007/s10439-011-0267-8

[25]Meng $\mathrm{H}$, Tutino VM, Xiang J,et al. High WSS or low WSS? Complex interactions of hemodynamics with intracranial aneurysm initiation, growth and rupture: toward a unifying hypothesis. AJNR Am J Neuroradiol.2014;35(7):1254-1262. http://dx.doi.org/ 10.3174/ajnr.A3558

[26]Menon A,Wendell DC,Wang H,et al. A coupled experimental and computational approach to quantify deleterious hemodynamics,vascular alterations, and mechanisms of long-term morbidity in response to aortic coarctation.J.Pharmacol Toxicol Methods.2012;65(1):18-28. http://dx.doi.org/

10.1016/j.vascn.2011.10.003

[27] Guzzardi D, Barker AJ, Ooij P,et al. Valve-related hemodynamics mediate human bicuspid aortopathy:insights from wall shear stress mapping. Journal of The American College of Cardiology.2015;66(8):892-900. http://dx.doi.org/ 10.1016/j.jacc.2015.06.1310

[28] Rinaudo A, Pasta S. Regional variation of wall shear stress in ascending thoracic aortic aneurysms. J Engineering in Medicine.2014; 228(6):627-638. http://dx.doi.org/ 10.1177/0954411914540877

[29] Burris NS, Hoff BA, Patel HJ, et al. Three-dimensional growth analysis of thoracic aortic aneurysm with vascular deformation mapping (VDM). Circ Cardiovasc Imaging.2018;11(8): e008045. http://dx.doi.org/ 10.1161/CIRCIMAGING.118.008045

[30] Koseki H, Miyata H,Shimo S,et al. Two diverse hemodynamic forces, a mechanical stretch and a high wall shear stress, determine intracranial aneurysm formation. Translational Stroke Research.2020;11(1):80-92. http://dx.doi.org/ 10.1007/s12975-019-0690-y

[31] Gs AK, Raj B, Santhosh KS,et al. Ascending aortic constriction in rats for creation of pressure overload cardiac hypertrophy model. Journal of Visualized Experiments, 2014;29(88):e50983. 
[32]Langille BL. Blood flow-induced remodeling of the artery wall. In: Flow-Dependent Regulation of Vascular Function, Bevan JA, Kaley G, and Rubanyi G,editors. New York: Oxford University Press, 1995.p.277-299.ISBN: 978-1-4614-7527-9

[33] Pierre B., Stéphane A., Susan L., Michael S. Mechanical identification of hyperplastic anisotropic properties of mouse carotid arteries. In: Proulx T. (eds) Mechanics of Biological Systems and Materials, Volume 2. Conference Proceedings of the Society for Experimental Mechanics Series. Springer, New York, NY. 2011; http://dx.doi.org/ 10.1007/978-1-4614-0219-0_2

[34]Gelidi de S,Bucchi A. Comparative finite element modelling of aneurysm formation and physiologic inflation in the descending aorta. Comput Methods Biomech Biomed Engin.2019; 22(15):1197-1208. http://dx.doi.org/ 10.1080/10255842.2019.1650036.

[35] Bjørnstad JL, Skrbic B, Sjaastad I.,et al. A mouse model of reverse cardiac remodelling following banding-debanding of the ascending aorta. Acta Physiol.2012;205(1): 92-102. http://dx.doi.org/ 10.1111/j.1748-1716.2011.02369.x

[36] Band W, Goedhard WJ, Knoop AA.,et al. Comparison of effects of high cholesterol intake on viscoelastic properties of the thoracic aorta in rats and rabbits. Atherosclerosis.1973;18(1): 163-171. http://dx.doi.org/ 10.1016/0021-9150(73)90127-5

[37] Guo XM,Lu X, Ren HM,et al. Estrogen modulates the mechanical homeostasis of mouse arterial vessels through nitric oxide. Am J Physiol Heart Circ Physiol.2006;290(5):1788-1797. http://dx.doi.org/ 10.1152/ajpheart.01070.2005

[38]Tuna BG, Ozturk N, U. Comelekoglu U,et al. Effects of organophosphate insecticides on mechanical properties of rat aorta. Physiol. Res. 2011;60(1):39-46. http://dx.doi.org/ 10.33549/physiolres.931941

\section{Figures}




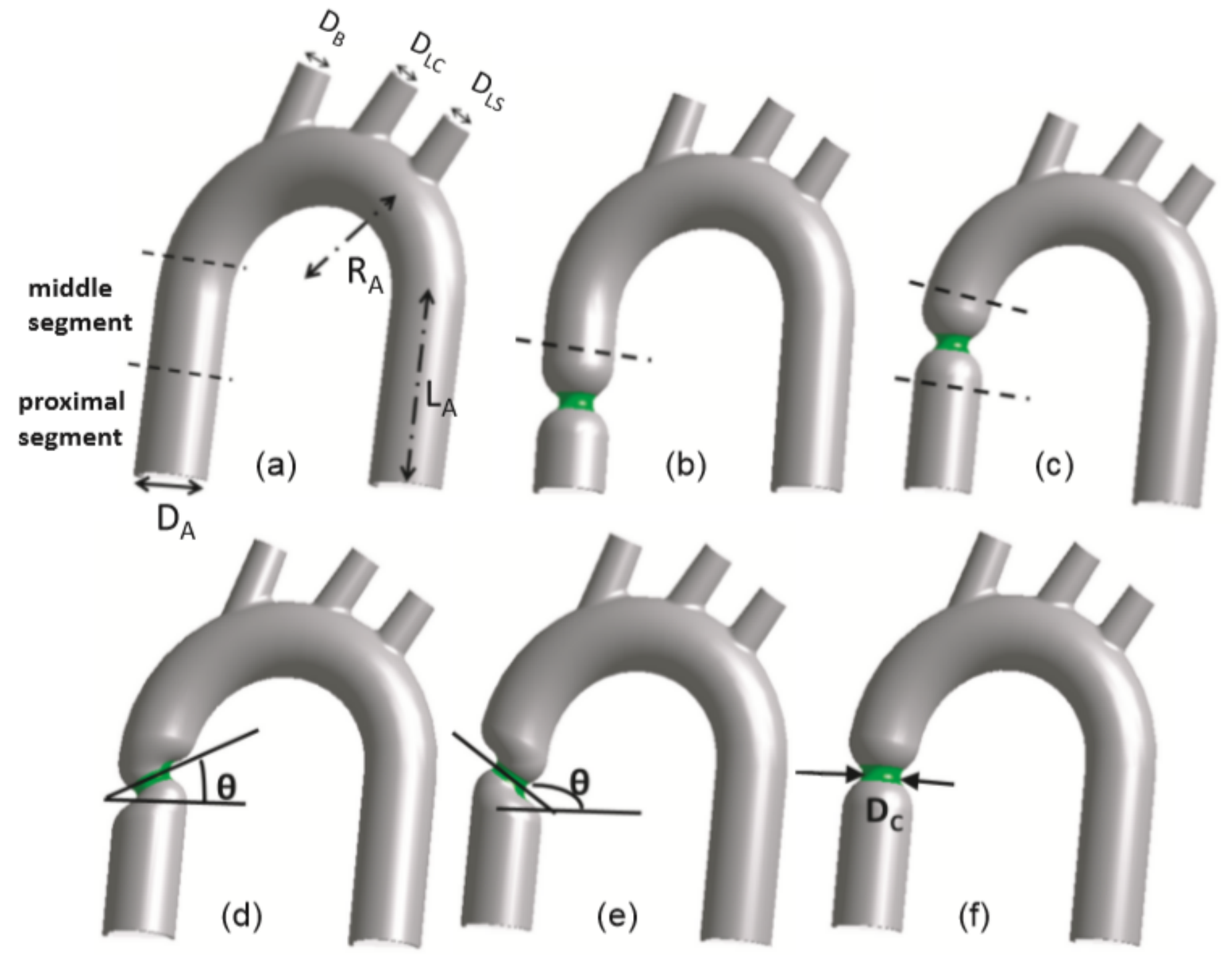

Figure 1

Banding models of rat aorta. (a)normal aorta model: DA: outer diameter of aorta, DB:outer diameter of BCA, DLC:outer diameter of LCCA, DLS:outer diameter of LSCA, RA:radius of aortic arch, LA: altitude of descending aorta (the same with ascending aorta) .(b)proximal segment banding model (c) middle segment banding model.(d) DC: outer diameter of banding ring .(d) $\theta$ : banding angle: acute angle.(e) $\theta$ : banding angle: obtuse angle. 


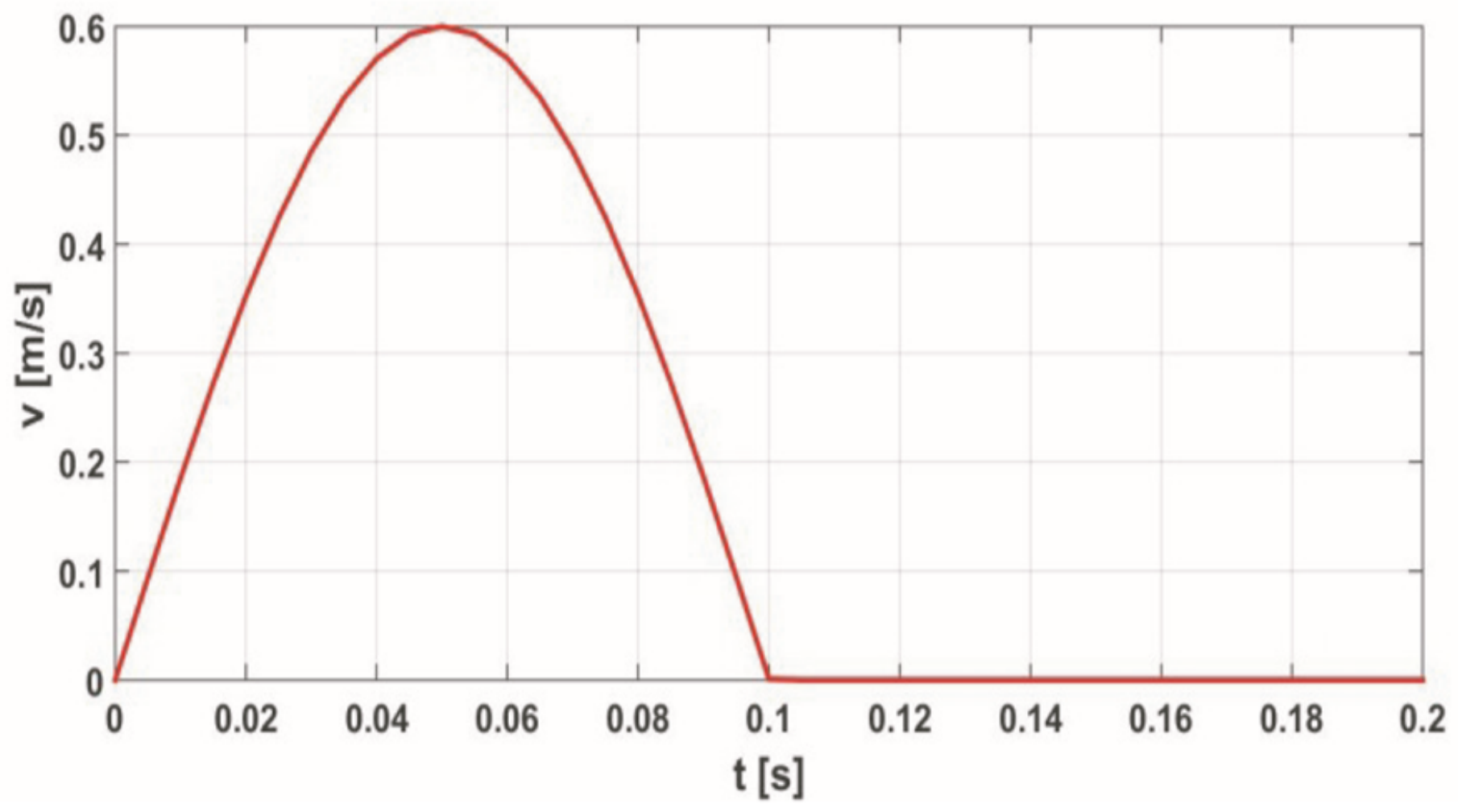

Figure 2

Longitudinal velocity at aorta root in one cardiac cycle 

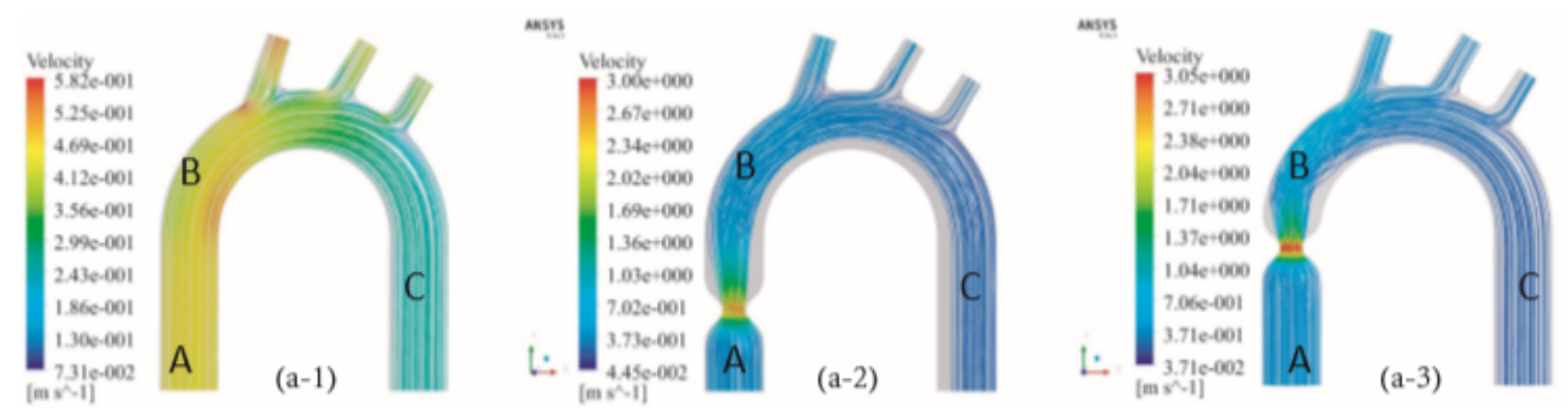

Ansrs

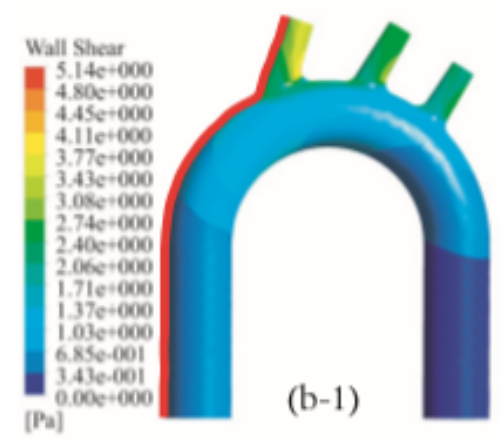
a*sก
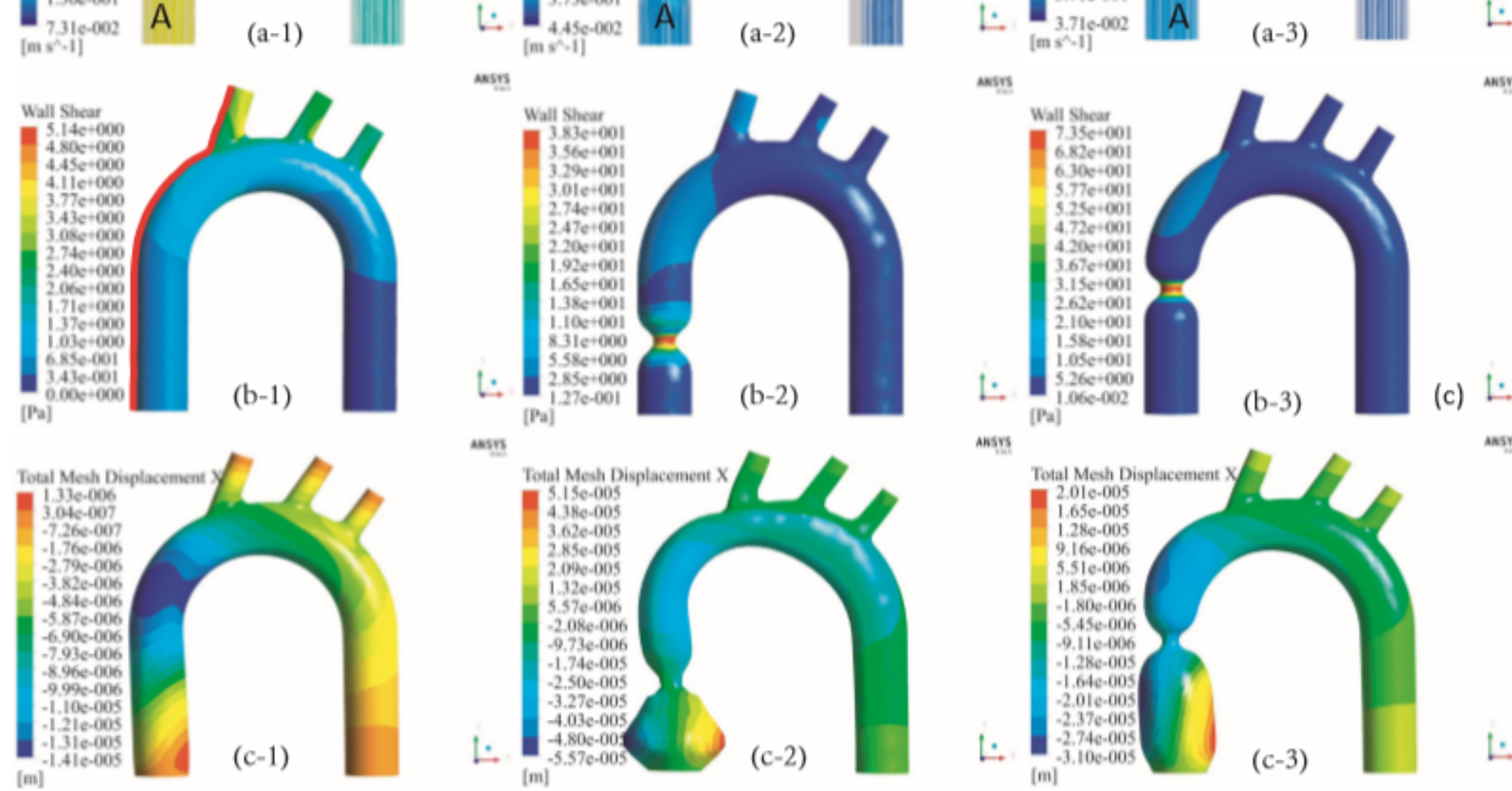

ansns

(c) $\stackrel{\circ}{\circ}$

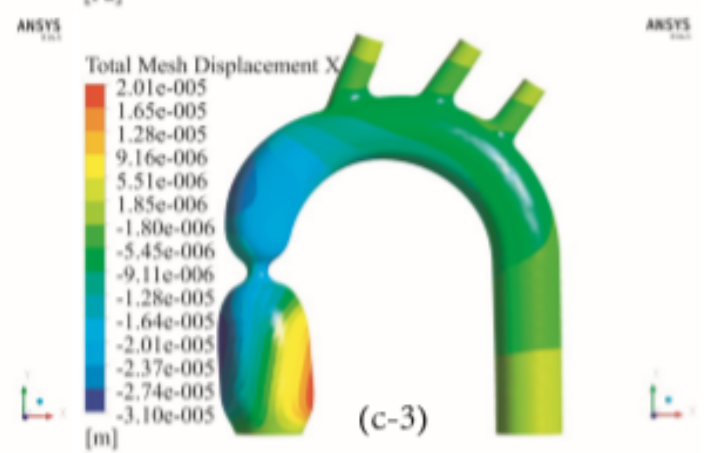

anss

\section{Figure 3}

Models with different banding altitudes .Velocity streamline of blood flow in (a-1) normal model, (a-2) proximal segment banding model, (a-3) middle segment banding model.WSS contours in (b-1) normal model, (b-2) proximal segment banding model, (b-3) middle segment banding model.Total mesh displacement contours in $\mathrm{X}$ direction with deformation in (c-1) normal model,(c-2) proximal segment banding model, (c-3) middle segment banding model.
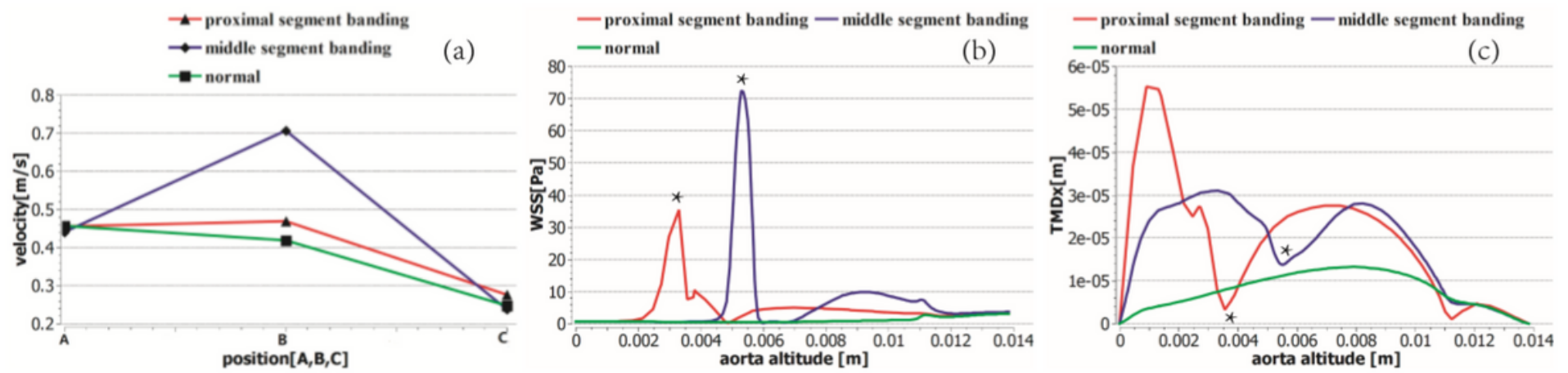
Comparison curves of different altitude models (a) average velocity in position A,B,C of three models,(b) WSS along the outer edge of ascending aorta of three models,(c)displacement (absolute value) along the outer edge of ascending aorta of three models, ${ }^{*}$ indicates the middle of banding ring.
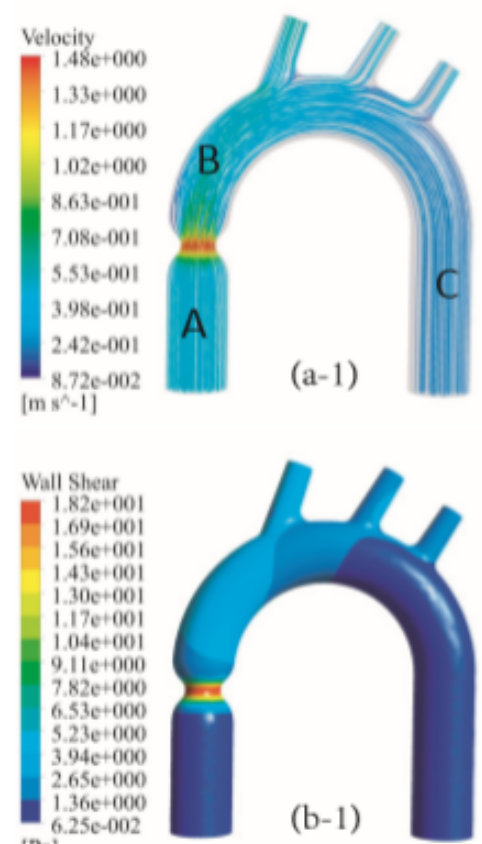

[Pa]
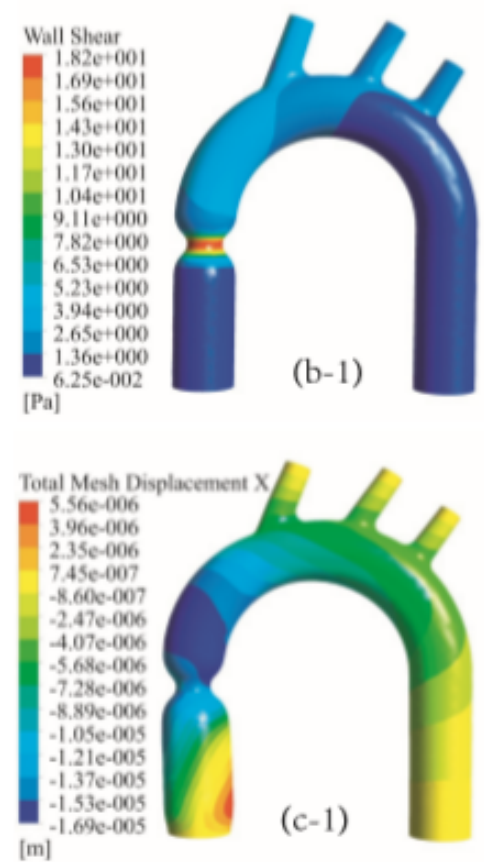
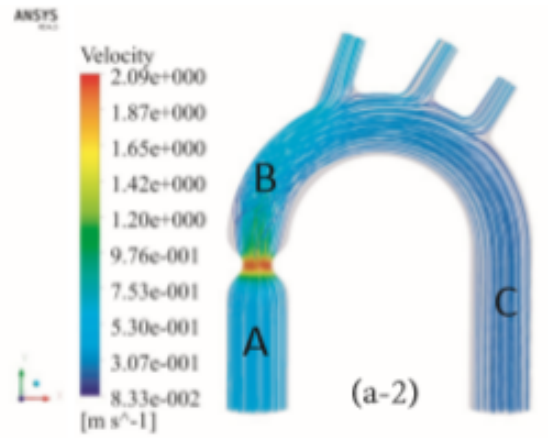

$$
\text { ass }
$$
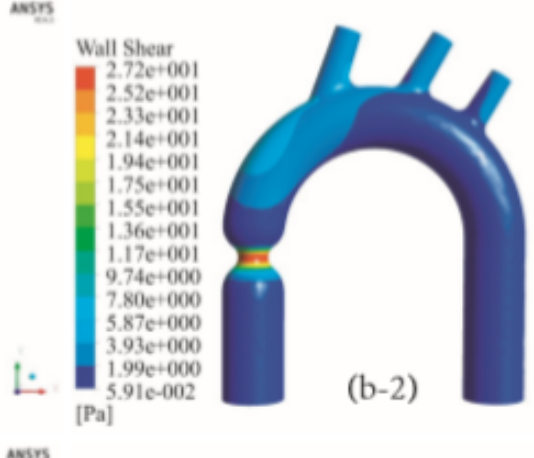

ansrs

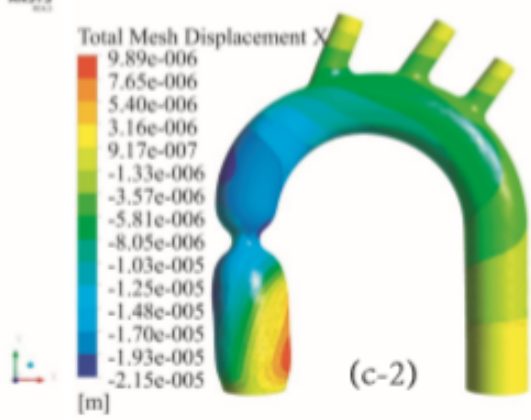

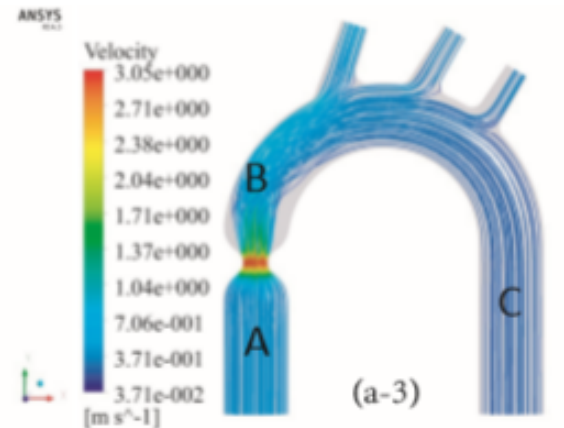

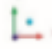

axsn

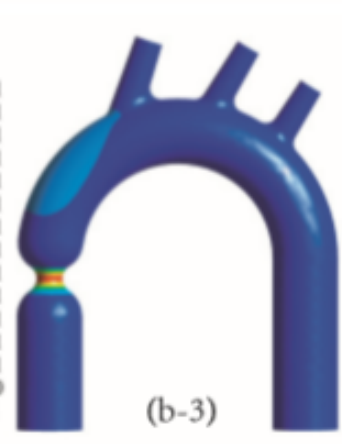

ansก

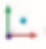

ass

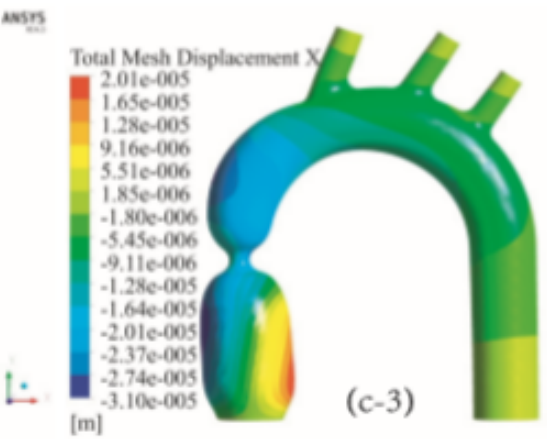

ass

\section{Figure 5}

Models with different banding severities .Velocity streamline of blood flow in (a-1) banding severity of $40 \%$, (a-2) banding severity of $50 \%$, (a-3) banding severity of $60 \%$.WSS contours in (b-1) banding severity of $40 \%$, (b-2) banding severity of $50 \%$, (b-3) banding severity of $60 \%$. Total mesh displacement contours in $X$ direction with deformation in (c-1) banding severity of $40 \%$, (c-2) banding severity of $50 \%$, (c-3) banding severity of $60 \%$.
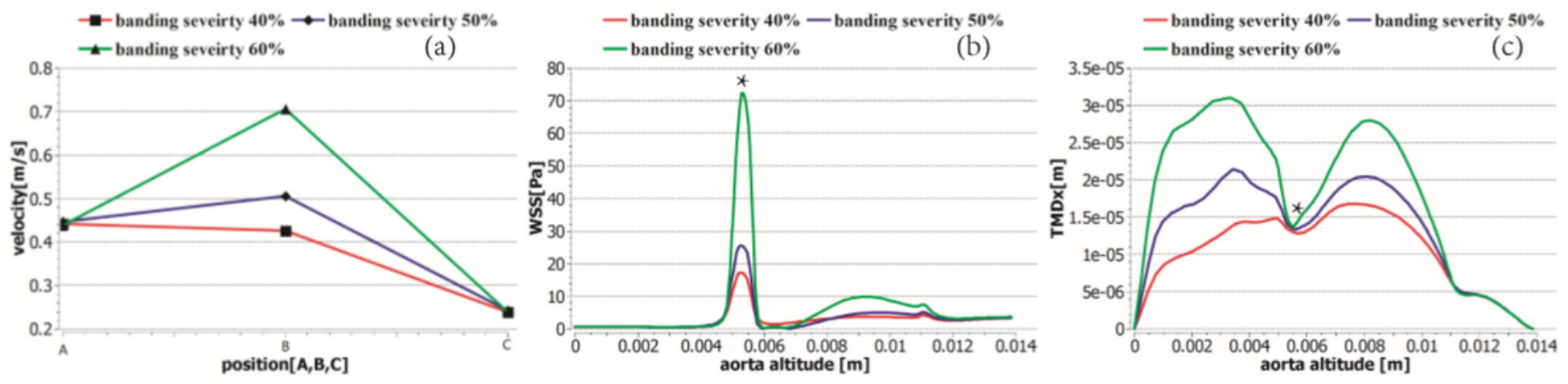


\section{Figure 6}

Comparison curves of different severity models.(a) average velocity in position $A, B, C$ of three models,(b) WSS along the outer edge of ascending aorta of three models,(c) displacement (absolute value) along the outer edge of ascending aorta of three models, * indicates the middle of banding ring
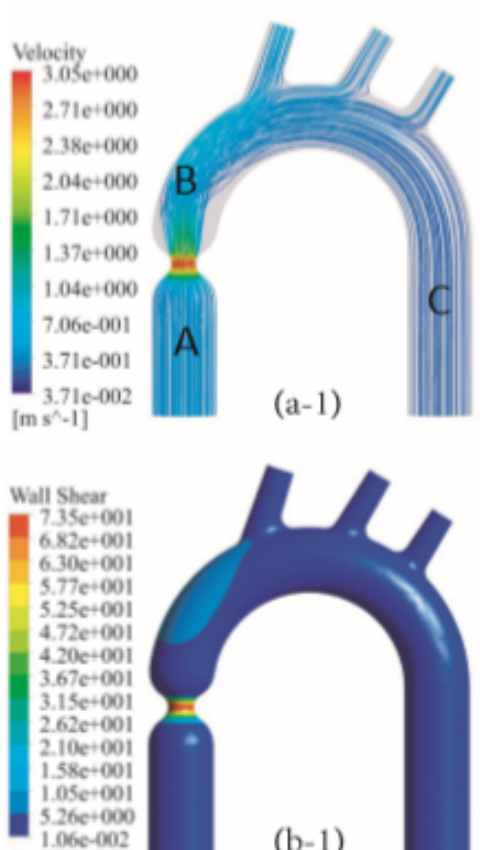
[Pa]
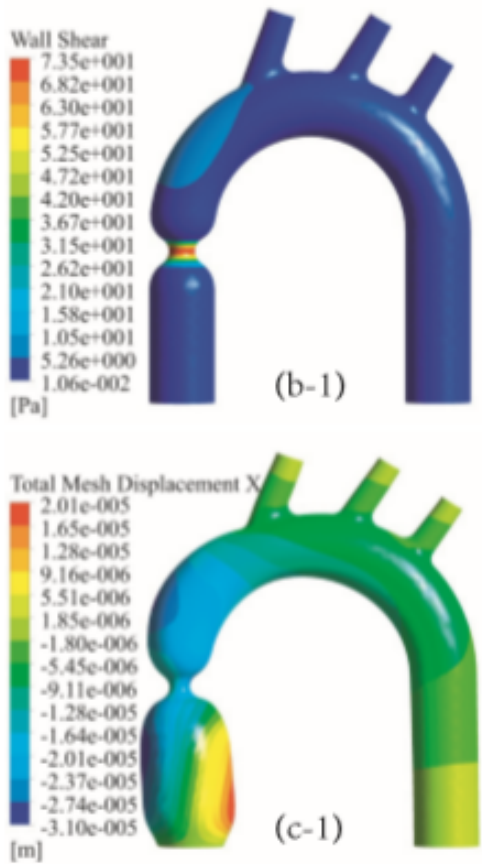

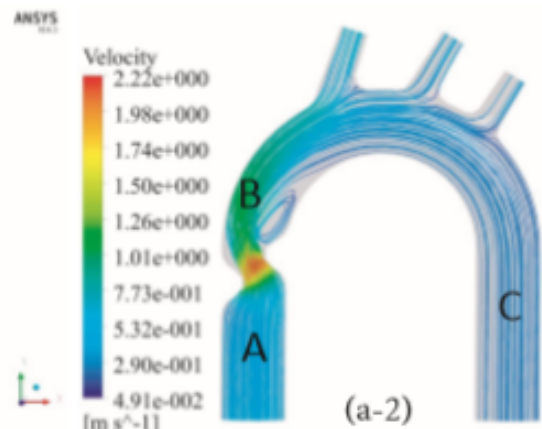

ansys
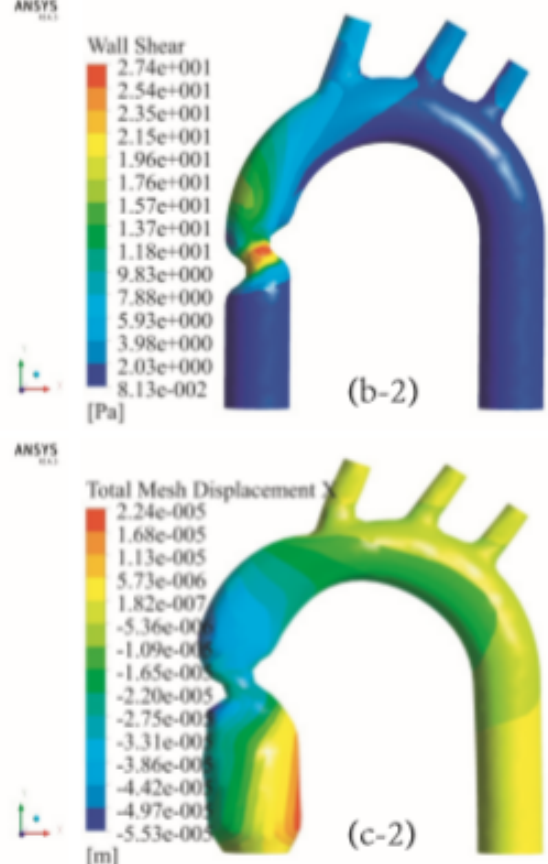

$2.74 \mathrm{e}+001$
$254 \mathrm{e}+001$

$2.54 \mathrm{e}+001$

$2.35 e+001$
$2.15 e+001$

$2.15 \mathrm{e}+001$
$1.96 \mathrm{e}+001$

$1.76 \mathrm{e}+001$

$1.57 \mathrm{e}+001$

$1.37 \mathrm{e}+001$

$9.83 e+000$

$7.88 \mathrm{e}+000$

$5.93 \mathrm{e}+000$

$3.98 \mathrm{e}+000$
$2.03 \mathrm{e}+000$

$2.03 e+000$
$8.13 e-002$

8.1

(b-2) ans?

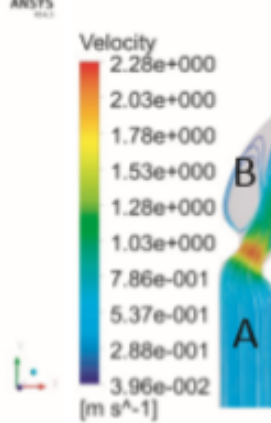

(a-3)

avss

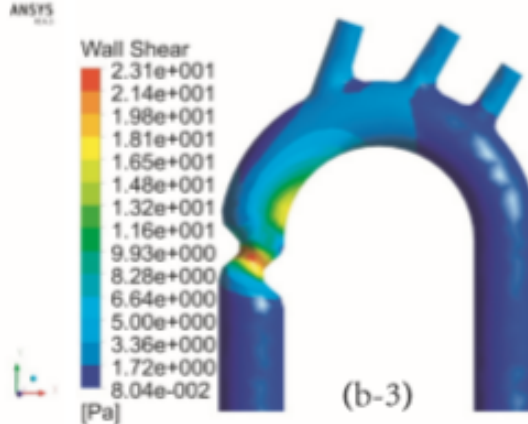

ans?

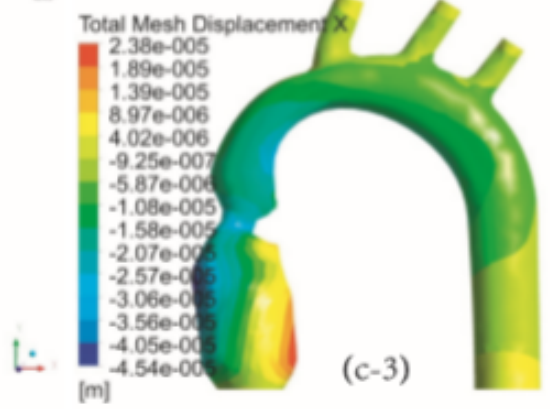

ansrs

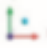

coss

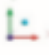

ass

เ

\section{Figure 7}

Models with different banding angles. Velocity streamline of blood flow in (a-1) banding angle of 0 degree, (a-2) banding angle of 30 degree, (a-3) banding angle of 150 degree.WSS contours in (b-1) banding angle of 0 degree, (b-2) banding angle of 30 degree, (b-3) banding angle of 150 degree.Total mesh displacement contours in $\mathrm{X}$ direction with deformation in (c-1) banding angle of 0 degree,(c-2) banding angle of 30 degree, (c-3) banding angle of 150 degree. 

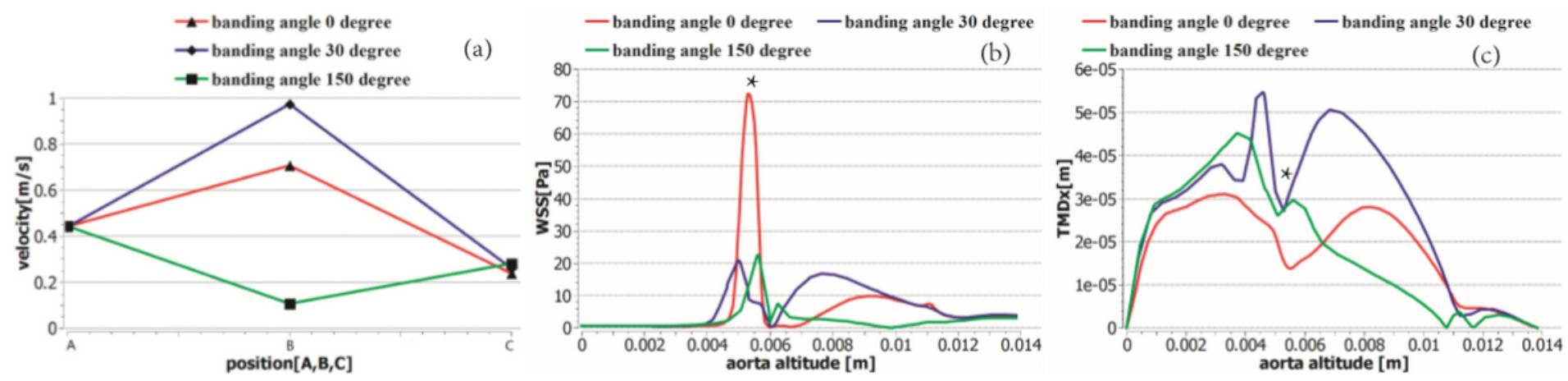

\section{Figure 8}

Comparison curves of different angle models. (a) average velocity in position A,B,C of three models,(b) WSS along the outer edge of ascending aorta of three models,(c) displacement (absolute value) along the outer edge of ascending aorta of three models, * indicates the middle of banding ring. 